\title{
El Biciactivismo en ciudades emergentes. El caso del Biciespacio en Posadas
}

\section{(4) Fernando Javier Rinaldi \\ Departamento de Geografía, Facultad de Filosofía y Letras, Universidad de Buenos Aires / Centro de Desarrollo y Territorio, Universidad Nacional de San Martín, Argentina.}

Recibido: 10 de octubre de 2017. Aceptado: 19 de enero de 2018.

\begin{abstract}
Resumen
A partir de entrevistas en profundidad y visitas a campo, el artículo describe la historia reciente del movimiento en favor del ciclismo urbano de la ciudad de Posadas, provincia de Misiones. En una primera sección se revisan los aportes teóricos producidos desde las ciencias sociales para abordar la movilidad ciclista, en una segunda sección se repasan aspectos relevantes de la historia del movimiento en favor de la bicicleta en Argentina y el mundo, para ya en la tercera describir las prácticas concretas de los biciactivistas posadeños.
\end{abstract}

\section{Bikeactivism in emerging cities. The case of Biciespacio in Posadas}

\begin{abstract}
Based on in-depth interviews and field visits, the article describes the recent history of the urban cycling movement in the city of Posadas, Misiones province. In a first section we review the theoretical contributions produced by the social sciences to approach cycling mobility, in a second section we review relevant aspects of the history of the movement in favor of the bicycle in Argentina and the world; and in the third section focuses in the history of the concrete practices of Posadas's bike activists.
\end{abstract}

\section{Introducción}

Las ciudades contemporáneas fueron planificadas y construidas para resolver el uso masivo e intensivo del automóvil particular. Este tipo de opción de movilidad genera enormes impactos negativos en los espacios públicos de las ciudades como grandes congestionamientos de vehículos en el tránsito en amplias franjas horarias y altos niveles de contaminación ambiental, solo por nombrar los más evidentes.

\section{Palabras clave}

Masa Crítica

Ciclismo

Movilidad

Activismo

Movimientos Sociales
Keywords

Critical Mass

Cycling

Mobility

Activism

Social Movements

Palavras - chave

Massa Crítica

Ciclismo

Mobilidade

Ativismo

Movimentos Sociais 
Pese a que en la mayoría de las actividades económicas existe una tendencia general a la reducción de emisiones de gases de efecto invernadero (GEI); en el caso del transporte automotor las emisiones tienden a aumentar a nivel global. Los automóviles promedio se diseñan para transportar cinco pasajeros con sus equipajes para distancias medias y largas; pero se utilizan de manera intensiva para viajes muy cortos, con pocos pasajeros y muy ineficientes desde un punto de vista energético. Además resultan objetos muy peligrosos en relación al peso y a la velocidad que pueden generar.

Una de las consecuencias más complejas de las altas tasas de motorización globales -y quizás menos abordada- es la disociación o suspensión de los atributos de ciudadanía en el ambiente vial y la creciente brecha de movilidad entre quienes acceden al automóvil y quiénes no. Esta brecha define un espacio público caracterizado por la inequidad vial (Rinaldi, 2014) y por altísimos niveles de siniestralidad -donde los más perjudicados son los grupos de menores ingresos-; además de generar barreras muy importantes para quienes se mueven con modos más seguros y sustentables como la bicicleta o el caminar (OMS, 2004).

En la actualidad existe una tendencia en planificación urbana a privilegiar la movilidad activa y el transporte público, sobre todo en las áreas centrales de las grandes ciudades del mundo como forma de combatir el cambio climático y pacificar el tránsito. En este contexto, la bicicleta ocupa un lugar central en el imaginario de esta tendencia. Amsterdam, Londres, Berlín, París y Copenhague son tomadas como referentes de ciudades europeas que están liderando un cambio hacia una movilidad más ciclable (ECF, 2014).

Latinoamérica no es ajena a esta tendencia. En grandes urbes como Bogotá, Rosario, Buenos Aires, México DF, San Pablo, Río de Janeiro o Santiago de Chile, pueden constatarse ejemplos de cambios a favor de los ciclistas impensables apenas diez años atrás. En ellas, pese a persistir un fuerte predominio del uso intensivo del automóvil, comienzan a aparecer, con sus más y sus menos, planes orientados a la movilidad sustentable, infraestructura ciclista y sistemas de bicicletas públicas con distintos alcances y coberturas (BID, 2015).

En este contexto surgen una enorme variedad de grupos y asociaciones de la sociedad civil para el fomento del ciclismo urbano no solo en las grandes ciudades argentinas, sino que también en muchas ciudades medias o "emergentes" de nuestro país ${ }^{1}$. La agenda de estos grupos incluye reclamos por infraestructura ciclista, por la contaminación ambiental que generan los combustibles fósiles, por mayor seguridad vial para pedalear y por la promoción de hábitos saludables.

Esta agenda constituye un posicionamiento político en el campo vial (Wright, 2010:6) definido no sólo en términos de la materialidad del viario sino como un conjunto de actores individuales e institucionales que disputan capitales sociales, culturales y económicos en función de sus intereses y necesidades dentro del espacio público. Consignas como "somos el tránsito" y "un auto menos" originadas en la Masa Crítica-presente en varias ciudades del país- son parte del repertorio común de estos colectivos.

Sin embargo, este significativo desarrollo de la movilidad ciclista y de los grupos biciactivistas en estas ciudades argentinas, sucede en un momento de gran crecimiento del parque automotor y de motovehículos. A partir de la expansión económica experimentada en los últimos quince años, personas provenientes de sectores medios y medios bajos han logrado acceder a un vehículo con motor -principalmente motovehículospara satisfacer sus demandas de movilidad, en parte porque la oferta de transporte público resulta cara o muy poco eficaz para resolver la movilidad cotidiana. Estos sectores que tradicionalmente eran usuarios de la bicicleta, hoy no encuentran buenos mecanismos de financiación para su compra, ni incentivos para su adopción. Esta verdadera paradoja, crecen los ciclistas pero también crecen la cantidad de autos y motocicletas en las calles; se acentúa en las ciudades emergentes del país (ANSV, 2011).
1. El Banco Interamericano de Desarrollo define como ciudad emergente en latinoamérica a aquellas que tienen entre 100.000 y 2.500 .000 habitantes y crecen económica y demográficamente por encima de la media nacional (BID,2016:3) 
En este artículo nos proponemos describir cómo los grupos de activistas de la bicicleta de las ciudades medias o emergentes de Argentina definen su ámbito de incidencia, qué actividades generan para ello y qué resultados obtuvieron. A partir de entrevistas en profundidad y visitas a campo del autor como observador participante, haremos foco en la historia reciente del movimiento en favor del ciclismo urbano de la ciudad de Posadas, provincia de Misiones. En una primera sección revisaremos los principales aportes teóricos producidos desde las ciencias sociales para abordar la movilidad ciclista. En una segunda sección repasaremos aspectos relevantes de la historia del movimiento en favor de la bicicleta en Argentina y el mundo, para ya en la tercera enfocarnos en relatar la historia de las prácticas concretas de los biciactivistas posadeños.

\section{De la circulación y el transporte a la movilidad sustentable}

El automóvil es el objeto central de la sociedad contemporánea. Desde la segunda posguerra hasta nuestros días se masificó su uso y producción y el hecho de poseer un auto se convirtió en un indicador de bienestar económico. Se habla de Fordismo y Toyotismo para definir períodos de la historia económica y todos los ministros de economía señalan la venta y producción de autos, junto a la cantidad de kilómetros de autopistas construidos, como un dato muy relevante para la economía.

André Gorz (1973), en su pionero ensayo La ideología social del automóvil sitúa la desigualdad relacionada con el uso intensivo del automóvil y la forma en que transformó el urbanismo de mediados del siglo XX. Planteaba que esta forma de movilidad materializaba un triunfo absoluto de la ideología burguesa al nivel de la práctica cotidiana y extendía la diferencia de clases a la velocidad y al medio de transporte.

Otros autores cuestionan la idea de desarrollo económico asociado al complejo automotriz y de las carreteras y sugieren que suele haber más aportaciones del Estado para sostenerlo que lo que obtienen las arcas Estado como reintegro social por su inversión (Sanz Alduán, 2015). A modo de ejemplo, Bahalla (2013) estimó que las pérdidas relacionadas con la siniestralidad vial se encuentran entre un 1.5\% y 2.9\% del PBI argentino, entre el $1.8 \%$ al 3.5\% en México y entre el 2.0\% al 3.9\% en Paraguay.

Desde hace al menos dos décadas, distintos especialistas, funcionarios y activistas comienzan a cuestionar este modo de pensar y construir la ciudad "desde atrás del parabrisas" (Sanz Alduan, 2005; Sebastián, 2006). Así surge el concepto de Movilidad Sustentable que sitúa la cuestión del tránsito en "algo más" que maximizar los volúmenes de transporte de cargas y personas a cualquier costo social y ambiental. Este paradigma va a poner un nuevo énfasis en valores como la accesibilidad, la equidad, la seguridad de las personas y en mitigar o erradicar las consecuencias negativas del transporte motorizado.

La sostenibilidad amplía el ángulo de visión de los problemas de la movilidad, haciendo hincapié en relacionar los desplazamientos con sus consecuencias ambientales, tanto las de carácter más local (contaminación del aire, ruido, ocupación de suelo fértil, fragmentación del territorio, etc.) como las de tipo global (cambio climático, biodiversidad, agotamiento de recursos, etc.). Pero, como es sabido, la sostenibilidad no es sólo ambiental sino también social y, por consiguiente, obliga a considerar también las consecuencias sociales del patrón de desplazamientos: salud, convivencialidad, autonomía de los diversos grupos sociales, etc. (Sanz Alduan, 2005:92).

Más recientemente surge la visión de "ciudades a escala humana", popularizada por el urbanista dinamarqués Jan Gehl, inspirador de intervenciones urbanas que buscan 
crear entornos que favorezcan lo sensorial y una nueva articulación entre forma urbana y vida cotidiana. Esta concepción crítica "la adopción del modelo mecánico de la ciudad moderna, donde la separación de las áreas funcionales y un sistema de movilidad donde el auto es el rey ha supuesto un retroceso para la escala peatonal de las ciudades" (Ciudades Sostenibles, 2013). La cercanía, la pluralidad de usos del suelo, la peatonalidad/ciclabilidad y la promoción de espacios urbanos compactos son los principales ejes que articulan esta mirada.

La creciente suburbanización es el fenómeno que caracterizó y sigue caracterizando el crecimiento de la mayoría de las ciudades del planeta, en especial las latinoamericanas y al cual se dirige de manera crítica esta visión. El tejido urbano tiende a lo discontinuo y disperso con áreas monofuncionales de baja densidad a las que hay que abastecer de infraestructura y servicios básicos. El paradigma de este tipo de urbanización es el barrio cerrado con su consecuente dependencia del automóvil.

Pero, como advierten Gutiérrez y Reyes (2017), la suburbanización en los países periféricos no es solo un feńomeno de las elites y las clases medias motorizadas, sino que incluye a sectores populares. Allí "la monofuncionalidad existe asociada con la precariedad urbana y con una movilidad vulnerable y no auto-intensiva" (Gutiérrez y Reyes, 2017:149). La inversión pública asociada a esta visión sobre lo sustentable y lo humano, como la recuperación de la bicicleta y la peatonalidad, no alcanzan a estos territorios que paradójicamente, son los que más necesitan de estas intervenciones ${ }^{2}$.

En las ciencias sociales se asiste a una suerte de giro o apertura hacia la movilidad consistente - grosso modo- en pasar de investigaciones inmóviles, donde los objetos de estudio se constituyen a partir de referencias estáticas como el barrio o el país; a considerar los viajes cotidianos de personas y objetos como legítimos problemas de estudio social y no solo como cajas negras con inicio en el punto A y final en el B (Sheller \& Urry, 2006).

Frente investigaciones desarrolladas para cuantificar y estratificar los desplazamientos de las personas entre distintos puntos y las razones de los mismos, en esta corriente el foco se pone en "recentralizar la experiencia corporal" de los sujetos durante el viaje (Sheller \& Urry, 2006). Esta valorización de la dimensión corporal en la movilidad abrirá una ventana teórica para los estudios relacionados con la movilidad activa en general y el uso de la bicicleta en particular.

La dimensión corpórea sensorial es central para analizar las prácticas cotidianas de ciclistas y peatones. El anterior paradigma basado en segmentar por tipo de viaje y por modo de transporte resulta insuficiente para comprender las motivaciones e itinerarios que desarrollan los ciclistas cotidianos. Como señalan Lorenzi Fernández y Ortega Botella (2016) los ciclistas urbanos no sólo se mueven en bicicleta por la ciudad, sino que viven la ciudad a través de ella. "La ciudad en sí misma no es objeto de ser entendido, sino las prácticas materiales y discursivas que en ella ocurren, donde la movilidad es una dinámica y no una categoría" (Lorenzi Fernández y Ortega Botella, 2016:4).

Este enfoque resulta aún más relevante cuando se examinan los principales factores que condicionan el cambio modal hacia la bicicleta. Existe un consenso en la bibliografía sobre la valoración que hacen los potenciales ciclistas en función de la distancia de sus itinerarios y de la falta de infraestructura específica (Pérez López, 2017). El temor al esfuerzo físico, al descontrol de fluidos y transpiraciones y la posibilidad de sufrir un siniestro, son dimensiones estrictamente corporales que necesariamente se deben abordar al momento de analizar y/o implementar políticas de fomento del ciclismo urbano.
2. Ejemplo de esto es la cuestión legal en la producción de veredas en el conurbano bonaerense argentino. La norma plantea que la producción de veredas es obligación de cada frentista; pero esta obligación suele ser incumplida y trae aparejado itinerarios discontinuos que incentivan a los peatones a caminar por la calle con el consiguiente riesgo vial (Notas del Autor. UPE AMBA, 2016). 
Más cercano a la biopolítica -en el sentido focaultiano- pero también en línea con este giro a la movilidad, se encuentran los aportes que están realizando la antropología, la sociología y la geografía a las disciplinas asociadas a la seguridad vial. Haciendo eje en el concepto de práctica social de Bordieu, Wright (2010) se enfoca en las prácticas cotidianas de los distintos actores del campo vial y concluye que para el caso argentino existe una tensión entre el sistema de prácticas viales y el sistema normativo. Las bocacalles, para este autor, son un ejemplo que el sistema vial se basa más en los flujos que en las normas de precedencia, con una primacía de los vehículos sobre los peatones. "Se trata de un sistema de presencia, que ignora el peso teórico de las normas escritas (que se observaría en un sistema de ausencia) valorando la dimensión performativa in situ del acto vial" (Wright, 2010:5).

Otra serie de trabajos cuestionan la concepción centrada en el automóvil imperante en las disciplinas en la seguridad vial. Herrera Gallo sostiene que intentar resolver la contradicción entre "sistemas viales inherentemente peligrosos" mientras simultáneamente se dedican las energías a perseguir infructuosamente el objetivo de lograr conductas perfectas resulta poco realista (Herrera Gallo, 2006:71). La materialidad de las infraestructuras viales en función de qué tipo de relación de poder favorece y cuál no, resulta una clave central de interpretación para estos trabajos.

De esta forma afirmaciones valorativas/punitivas como la de "peatones imprudentes", "conductores irracionales" o "los ciclistas no respetan nada" pasarán a tener una luz diferente si se las enfoca en función de categorías como la equidad o la vulnerabilidad. El hecho de que las infraestructuras viales favorezcan la circulación del transporte automotor, permite a los conductores obtener ventajas que ponen a los demás actores viales en una situación inequitativa; ya que por más que los peatones y ciclistas sepan que tienen derecho de paso, muy pocos desearán ejercerlo frente a un vehículo que avanza sin frenar en la bocacalle (Rinaldi, 2014:3).

Una última vertiente de este "giro hacia la movilidad" producido en las ciencias sociales está relacionada con la construcción del campo vial ya no como un sistema de prácticas y sujetos; sino como un ámbito específico donde distintos actores, sean públicos, privados o provenientes de la sociedad civil; disputan, acuerdan y perfilan intervenciones en y sobre lo vial en una misma red de relaciones de interés e incidencia. Este interés, si bien incluye la posibilidad de obtener alguna ganancia personal, debe ser entendida como una noción más flexible y acotada a conseguir alguna posición material, simbólica, técnica o discursiva -a veces más aceptable qué óptima- desde donde continuar y/o profundizar la incidencia sobre el propio campo (Moro, 2000).

Proponemos esta definición de campo vial para ampliar la propuesta por Wright (2010) porque nos permite situar el conjunto de determinadas prácticas o performances de los sujetos en la calle, en relación con los procesos históricos y económicos que determinaron la construcción material del viario y son la condición necesaria de esas prácticas. En ese sentido, trabajos como los de Ballent (2005) sobre el entramado de actores e instituciones que perfilaron en sus inicios la configuración de la red vial argentina, el de Piglia (2014) sobre la articulación del Automóvil Club Argentino con el Estado para la ampliación de la red vial o el de Oppezzo (2008) sobre las asociaciones de familiares de víctimas de siniestros viales en Argentina son algunos ejemplos de esta creciente literatura que aporta herramientas relevantes para comprender las políticas relacionadas con la movilidad ciclista contemporánea y la dinámica de los movimientos en favor de la bicicleta.

Cómo señala Moro "la capacidad técnica por sí sola no garantiza la efectividad de las políticas, se necesita también llegada a referentes, instituciones o espacios significativos que funcionen como vínculo y vehículo de acciones planificadas" (Moro, 
2000:119). Tal es el caso de las organizaciones de familiares y víctimas de siniestros viales, que construyeron "masa crítica" para la creación de la Agencia Nacional de Seguridad Vial (ANSV) y son generadores de mensajes de sensibilización, vistos como mucho más legítimos que los organismos estatales encargados de la seguridad vial (Oppezzo, 2008) .

La asociación de determinados valores cívicos o políticos a un modo de transporte sobre otro también puede ser una herramienta de legitimación para las intervenciones materiales necesarias para la expansión del modo. En el caso del diseño de la red vial argentina a principios de los años 30 del siglo pasado la idea de malla y no de abanico, constituyó una imagen de integración territorial sumamente poderosa y desafiante de la hegemonía del ferrocarril como modo de transporte, más allá de consideraciones técnicas, económicas o geográficas (Ballent, 2005:116); que legitimaron el papel y la relevancia de la naciente Dirección Nacional de Vialidad y del transporte automotor.

Promover el uso de la bicicleta en la ciudad, es principalmente un desafío político ya que la mayoría de las ciudades han enfrentado o enfrentan fuertes resistencias sobre la potencialidad que tiene la presencia de este vehículo en el espacio vial (BID, 2015). Por ello, resulta relevante analizar los discursos y las prácticas concretas con que el Estado, el sector privado y la sociedad civil afrontan el desafío de legitimar intervenciones cicloinclusivas y que espacio ocupa la participación ciudadana en estas políticas. En la siguiente sección desarrollaremos un brevísimo repaso desde una perspectiva histórica del cicloactivismo mundial y argentino.

\section{Bicicletas, políticas ciclo inclusivas y colectivos sociales. El ciclismo urbano como una militancia social}

A finales del siglo XIX existían, principalmente en Europa, clubes y organizaciones ciclistas dedicadas al fomento de la actividad deportiva y estaban principalmente conformadas por miembros masculinos de las élites políticas y económicas (Gray y Watson, 1978). Estos primeros ciclistas eran vistos como una verdadera amenaza social debido a su mayor velocidad frente a los carros tirados a caballo y los peatones que circulaban por el espacio público, por lo que surgen numerosos decretos y ordenanzas para regular su tránsito.

Para principios del siglo XX, las mujeres sufragistas europeas y estadounidenses -llamadas así porque comenzaron a reclamar su derecho a votar en las elecciones- encontraron en la bicicleta no solo una forma de movilidad individual sin chaperones masculinos, sino que hicieron de este vehículo un símbolo clave en la lucha por sus derechos.

La bicicleta además revolucionó la vestimenta femenina, impuso el fin del corsé y posibilitó el uso de pantalones. Frances Willard (1839-1898), educadora y militante norteamericana por la igualdad, señaló que el sencillo hecho de que las mujeres ciclistas resultaran tan aptas para pedalear y reparar sus bicicletas como cualquiera de los ciclistas varones era una demostración práctica, clara e indiscutible de la igualdad entre los géneros. Con el agregado de que el ciclismo ofrecía una nueva conciencia corporal a partir de una actividad física al aire libre en mujeres cuyas vidas estaban sesgadas a lo doméstico.

No será hasta mediados de los años 60' del siglo XX que la bicicleta comenzará a ser vista como objeto antagonista a la masificación del automóvil. Las llamadas bicicletas blancas fueron una de las ideas más reconocidas del Provo, movimiento pionero en la crítica ambiental al capitalismo en los Países Bajos. La acción de las bicicletas blancas ${ }^{4}$
3. Algunos ejemplos destacados de estas organizaciones son: Madres del Dolor, Conduciendo a Conciencia, Padres en la Ruta y ACTIVASS. Existe un comité de Justicia y Derechos Humanos dentro del Consejo Consultivo de la ANSV que las nuclea y las reconoce como interlocutores válidos de las acciones del Estado en materia de seguridad vial.
4. En la actualidad las bicicletas blancas son hitos viales que señalan el lugar donde un ciclista falleció víctima de algún siniestro. Véase http://ghostbikes.org/ 
consistía en la puesta a disposición de bicicletas pintadas de ese color, provistas por los propios integrantes del movimiento, para que fueran usadas sin cargo de alquiler por los habitantes de Ámsterdam como forma de protesta y resistencia contra la propiedad privada, la cultura del automóvil y la contaminación (Carlsson et al., 2012).

Durante la crisis del petróleo, a principios de los años 70 del siglo pasado, la bicicleta comienza a ser asociada a la mitigación de las consecuencias ambientales del automóvil por los nacientes grupos ecologistas y se convertirá en un símbolo de autonomía e independencia energética para numerosos grupos vinculados con la izquierda radical y la contracultura en los países centrales. En este contexto el presidente de Estados Unidos Jimmy Carter presenta al congreso una ley sobre la política nacional energía que incluye un presupuesto específico para promocionar la bicicleta como forma de transporte. Para este momento en los países bajos comienza a surgir una revalorización de la bicicleta como forma de movilidad asociada a la construcción de infraestructura específica.

En 1992, más precisamente el último viernes de septiembre, un grupo de ciclistas de San Francisco llevó a cabo un paseo ciclista sin líderes que se convirtió en la primer Masa Crítica. La idea de masa crítica aplicada a la cultura ciclista aparece en el film sobre bicicletas The return of the Scorcher (Ted White, 1992). Allí George Bliss describió que los ciclistas de Shangai se congregan en las esquinas hasta lograr un grado suficiente de "masa crítica" para atravesar las grandes avenidas de la ciudad. Empezó como una celebración mensual, y en los años siguientes el concepto de coincidencia organizada se extendió alrededor del mundo.

Es un ejemplo fantástico de acción colectiva que escapa al tradicional cuadro que otorga todo el crédito a algún gran hombre o individuos brillantes como como la fuente de los movimientos sociales. El concepto de pedalear en conjunto en masse con final abierto fue lo suficientemente amplio para ser adaptado en muchas maneras durante las décadas de pasadas, desde alterar la estructura de la bicicleteada recreativa organizada, al uso del estilo Masa Crítica para una amplia gama de temas y campañas de activismo político (Carlsson, 2012:9. Trad. propia).

La Masa Crítica implica un desafío de carácter festivo pero antagónico a la hegemonía del automóvil y el petróleo en los sistemas de transporte y a sus consecuencias en la urbanización y el modo de vida de la ciudad (Lopez León, 2016:56). Pero este desafío en clave celebratoria no está exento de conflictos, tratamiento estigmatizante por parte de los medios de comunicación, enfrentamientos con la policía y las autoridades o directamente atropellos masivos de ciclistas por parte de automovilistas violentos. De hecho, el Foro Mundial de la Bicicleta (FMB) nació como una respuesta del movimiento ciclista global frente al atropellamiento intencional que sufrieron los ciclistas participantes de la Masa Crítica de Porto Alegre el 25 de febrero de 2011 por parte de un conductor.

El auge de la Masa Crítica en numerosas y disímiles ciudades del mundo hacia finales del siglo XX y a principios del nuestro, permitió que muchas personas y colectivos sociales del mundo hagan de la bicicleta y sus pedaleos cotidianos una forma de expresión político-cultural. En muchas ciudades se reparten patentes o matrículas artesanales para bicicletas con leyendas que identifican a los ciclistas maseros. Este reconocimiento de la participación de los objetos en la vida social, ejemplificado en la consigna "Autos no; Bicis si" coreada en las coincidencias argentinas, advierte sobre la artificialidad de escindir lo social de lo material y de cómo en el ambiente vial se produce una mimesis objeto/sujeto (Diz, 2016).

Internet y las redes sociales están estrechamente relacionadas con la difusión global y local de la Masa Crítica. Como señala López León (2016), el uso de las nuevas 
tecnologías permite reducir los costos de participación a través de prácticas de activismo lúdico; "por lo que un mayor número de activistas pueden recuperar su papel de confrontación, en el caso del ciclismo urbano, de reivindicación, y también ofrece la oportunidad de no centralizar los esfuerzos del movimiento en pocas personas" (López León, 2016:55).

Pese a los múltiples entrecruzamientos de numerosos ciclistas deportivos y urbanos; nunca pudo construirse una relación sinérgica entre el ciclismo deportivo y el de movilidad como si resultó en el caso del automovilismo argentino (Ballent, 2005; Piglia, 2014). Inclusive se advierte cierto recelo entre los practicantes de uno u otro género ejemplificado en comentarios como "no es deporte, es transporte" o la siempre inestable apropiación de la palabra "ciclismo" para definir la movilidad en bicicleta por su connotaciones deportivas.

En nuestro país la Masa Crítica aparece en el año 2008 en Buenos Aires y rápidamente se propaga a las principales ciudades del país. En un recuento que hizo este autor utilizando las redes sociales surgieron Masas en al menos diez ciudades importantes de Argentina, entre los años 2011 y 2015. Hasta la irrupción de la Masa existían grupos y asociaciones de ciclistas no competitivos, pero sus intereses estaban vinculados a lo recreacional y al cicloturismo. Solo la Asociación de Ciclistas Urbanos (ACU) incluía en sus acciones cuestiones relacionadas con la seguridad vial del ciclista y la promoción de la bici como forma de transporte ${ }^{5}$.

Entre setiembre y octubre del 2010 parece ser el momento en el que la Masa de Buenos Aires dejó de ser de cientos de ciclistas para pasar a ser de miles. Logró picos de masividad entre 2011 y 2014 y hoy la participación se redujo en cantidad de participantes pero también se estabilizó. Lograr algún indicador objetivo de participación en un evento de estas características supone algunos desafíos metodológicos que sólo enunciaremos. ¿Cómo medir la participación de un evento autoconvocado, en movimiento y sin final fijo? ¿Es relevante intentar "contar" a los ciclistas o se puede medir el impacto en tiempo de paso del pelotón por un hito dado? La Masa también ofrece distintas dimensiones de estudio, desde los “tapones" (bloqueos al tránsito en las bocacalles producidos por ciclistas "espontáneos") hasta las reacciones de los automovilistas que se cruzan -literalmente- con el evento.

La Masa Crítica en Argentina se constituyó en un verdadero laboratorio para un nuevo tipo de activismo político y cultural que combina aspectos ecológicos, políticos, contraculturales, comunitarios, de género y viales. Entorno a ella aparecieron blogs, fanzines, programas de radio entre otras iniciativas que incluyen a la bici pero a la vez la exceden. Un ejemplo muy particular de esto son los talleres autónomos de ciclomecánica popular, conocidas en los países centrales como BikeKitchen o Cicloficcinas. La Fabricicleta -hoy desactivado-, Chela en Buenos Aires o el Taller de Suipacha en Córdoba son algunos ejemplos locales. Se trata de espacios de base donde se intercambian partes y saberes técnico-mecánicos para reparar y restaurar bicis. Allí la bicicleta representa no solo un medio de transporte, sino que se constituye en un vehículo que, frente a los dictados de la sociedad de consumo, potencia la libertad, la autonomía personal y la autogestión.

En 2016 se conformó Argentina en Bici, una organización federal que nuclea a la mayoría de los grupos de activismo ciclista con el objetivo de demandar más y mejores infraestructuras específicas, datos oficiales de participación modal, políticas cicloinclusivas y cambios en la normativa vial. De este primer encuentro participaron representantes de 16 colectivos ciclistas de todo el país, principalmente de las ciudades emergentes de la Argentina (Ver Cuadro 1). En su gran mayoría estos colectivos surgieron de las Masas Críticas locales y su antigüedad promedio ronda los cinco años ${ }^{6}$.
5. La A.C.U. surgió de las masivas marchas de "Amigos de la Bicicleta" del año 1997 auspiciadas por empresarios del rubro y de las que participaron cientos de ciclistas. Vinculada en sus inicios al cicloturismo y al ciclismo recreativo, fue girando hacia la movilidad ciclista cotidiana. En 1999 logró convocar a una audiencia pública en la legislatura de CABA sobre ciclismo urbano en el año 1999. Hoy integra el Consejo Consultivo de la ANSV y realiza actividades de incidencia y capacitación vial.

6. Al cierre de este artículo (setiembre de 2016) se realizaba el $1^{\circ}$ Foro Argentino de la Bicicleta y el segundo encuentro de Argentina en Bici al que se incorporó el Biciespacio de Posadas. 
Cuadro 1. Colectivos Ciclistas fundantes de Argentina en Bici. Fuente: Documento Fundacional de Argentina en Bici (2016)

\begin{tabular}{|l|l|}
\hline Organización & Ciudad \\
\hline Anda la bici & Salta, Salta \\
\hline A Rodar Rosario Cicloturismo & Rosario, Santa Fe \\
\hline Asociación de Ciclistas Urbanos & C.A.B.A. \\
\hline Bicicletas por la vida & San Carlos de Bariloche, Río Negro \\
\hline Bicicultura Funes & Funes, Santa Fe \\
\hline Biciurbanos & Córdoba, Córdoba \\
\hline Ciclofamilia & C.A.B.A. \\
\hline Ciclistas Urbanos Confluencia & Cipolletti, Neuquén \\
\hline Mujeres Bici-bles & Salta, Salta \\
\hline Ramirez Pedalea & Gral. Ramírez, Entre Ríos \\
\hline Rosario en Bici & Rosario, Santa Fe \\
\hline Santa Fe en Bici & Santa Fe, Santa Fe \\
\hline Taller Popular de Ciclomecánica de Río Ceballos & Río Ceballos, Córdoba \\
\hline Taller Popular de Ciclomecánica “Suipacha” & Córdoba, Córdoba \\
\hline Taller Popular de Ciclomecánica “La Luna” & Córdoba, Córdoba \\
\hline Tres Arroyos Movilidad Sostenible & Tres Arroyos, Buenos Aires \\
\hline Viking Bike Academy & C.A.B.A. \\
\hline
\end{tabular}

Este diverso movimiento ciclista tiene su contraparte incipiente en los gobiernos locales de las ciudades del país y en el Ministerio de Transporte de la Nación, que han desarrollado organismos específicos para la promoción de la movilidad en bicicleta. Las manifestaciones comunes de políticas en favor de la bici en Argentina son la implementación de sistemas de bicicletas públicas y la ampliación de las redes de bicisendas y ciclovías; iniciativas que a su vez resultaron en un incentivo para la incorporación de nuevos ciclistas de movilidad.

Los gobiernos de Buenos Aires y Rosario llevan más de diez años sosteniendo políticas ciclo inclusivas con resultados diversos que no es propósito de este artículo analizar. Solo señalaremos que tuvieron la virtud de enfrentar las resistencias iniciales que estas intervenciones producen en la población y situar a la bicicleta como un vehículo apto para realizar itinerarios urbanos. Esto a su vez abrió una ventana de posibilidad a los grupos de activistas para su propia constitución como sujetos de políticas públicas, porque hace de la cuestión de la movilidad ciclista algo factible, que goza de cierto consenso y principalmente, que puede ser solucionable dentro del status quo (Moro, 2000:139).

\section{Pedaleando por Posadas}

En esta sección repasaremos la historia, principales actividades, logros y la evolución de las formas organizativas del biciactivismo de la ciudad de Posadas, Misiones; desde la Masa Crítica al colectivo BiciEspacio. Para ello utilizaremos distintas fuentes - artículos periodísticos, publicaciones en internet, programas de radio sobre ciclismo urbano y redes sociales entre los años 2012 y 2017-, entrevistas en profundidad con dos referentes clave del biciactivismo de la ciudad y una visita a campo del autor como observador participante el 9/06/2016 -invitado por el Biciespacio y el concejal de Posadas Fernando Meza- en el marco de la celebración del día de la educación vial. 
Posadas es la capital de la provincia de Misiones, ubicada en el extremo noreste de la Argentina. Para el censo 2010 (INDEC, 2010), el departamento Capital contaba con 324.756 habitantes y registró un crecimiento intercensal del 14,2\% (2001-2010), explicado en parte por el crecimiento de localidades como Garupá y Candelaria, que actualmente constituyen lo que suele nombrarse como el "Gran Posadas". Este crecimiento en clave suburbana tuvo su correlato en un crecimiento del parque automotor de aproximadamente un 35\% durante el mismo período.

Lorenzi Fernández y Acero (2016) definen cuatro condiciones para una ciudad en transición hacia una movilidad ciclista: (a) las políticas urbanas de movilidad y las de ordenación territorial, (b) los movimientos sociales relacionados con la bicicleta, (c) las cualidades urbanas y medioambientales -orografía, climatología, red viaria, infraestructuras, etc.- $y$ (d) las configuraciones sociales, actitudes y percepciones ante la seguridad, la comodidad y la eficiencia. La promoción de la movilidad en bicicleta se presenta para muchos funcionarios, planificadores y activistas de Posadas como una forma de mitigar las consecuencias de mayores tasas de motorización producto de la suburbanización reciente.

Posadas cumple con las condiciones (b) y (d), pero existe una percepción negativa en algunos actores políticos e institucionales respecto de la (c) que los biciactivistas refutan. Posadas tiene muchas cuestas y altas temperaturas medias; lo que podría constituir un obstáculo "porque que a la gente no les gusta transpirar". Pero en la visión de los activistas es el miedo al viario y la falta de infraestructuras el principal obstáculo a superar, más que las particularidades climáticas u orográficas. En lo referido a la condición (a), si bien Posadas construyó algunas ciclovías, el estado actual del viario específico dista mucho de lo óptimo y no llega a convertirse en una red (ver siguiente sección).

En este contexto de alta motorización y crecimiento suburbano, propio de una ciudad emergente surge la primer Masa Crítica de Posadas en abril de 2012. La Masa posadeña contiene las principales características de este movimiento global: ausencia de liderazgos o representantes oficiales, un punto de encuentro y una fecha fija, final abierto y un tono abiertamente festivo (Carlsson et al., 2012). A diferencia de muchas ciudades del mundo, donde la Masa sucede en un día hábil; el encuentro fue fijado en Posadas para los primeros domingos de cada mes, como en Buenos Aires. La Masa porteña fue una de las principales influencias que incentivaron la conformación en Posadas.

Todos los biciactivistas consultados señalan que si bien existían grupos de usuarios que se ocupaban del ciclismo urbano con anterioridad a la aparición de Masa Crítica - como la Asociación de Ciclistas Urbanos de Posadas-, este movimiento aportó otra perspectiva con respecto al uso de la bicicleta en Posadas al incorporar dimensiones culturales o de género entre otras, pero sin perder los reclamos propios de la bicicleta dentro del campo vial. En palabras de Ana Espinoza, integrante del primer núcleo masero y reconocida periodista local, el evento "tuvo en cuenta la diversidad de las personas que andaban por la ciudad y, más que nada, incorporó a los jóvenes”.

En estos cinco años, la Masa Crítica posadeña tuvo dos características positivas para los ciclistas entrevistados. Primero, la permanencia; la Masa logró conformarse todos los primeros domingos de mes de manera ininterrumpida, aunque fueran muchos o pocos los ciclistas autoconvocados. El segundo es que en la Masa Crítica se logró sensibilizar a los participantes sobre las implicancias políticas, sociales y culturales de la bicicleta en el campo vial y articular un discurso común "para que haya más conciencia sobre el uso de la bici en nuestra ciudad" (Ana E.). Si bien la Masa alcanza picos de participación asociados a algún evento particular (aniversarios, día de la tierra, recitales) la participación media oscila entre las 60 y 20 personas. 
Como en otras ciudades de la Argentina, la Masa Crítica fue la chispa que encendió distintas iniciativas de activismo ciclista en Posadas como fiestas rodantes, un primer colectivo de incidencia ciclista y un programa de radio pionero en ciclismo urbano. En palabras de Paola T.:

Es una forma de militancia alegre y una manera de sentirse respaldado, ése fueron mis primeros sentimientos que tuve en la Masa Crítica, el de pedalear sin miedo. Salimos a pedalear con nuestros objetivos bien claros, pero salimos desde la alegría y eso lo ven nuestros amigos, los periodistas, la gente. Además nos ha permitido crear una causa ciclista en Posadas que nos aportó a los cicleros mucha esperanza

Si bien durante 2012 la Masa como colectivo fue invitada a participar de distintos eventos autogestivos y también estatales, para 2013 cuatro personas del núcleo fundador deciden crear el grupo Usa la bici, Pedalea la vida como manera de encarar la militancia ciclista respetando el principio de no liderazgo, sin voceros oficiales y apartidario propios de la definición más ortodoxa de la Masa Crítica.

Este colectivo realizó distintas actividades de promoción entre las que se destaca la instalación de la primer bicicleta blanca de Argentina en memoria de Juan Ramón Gómez, un ciclista que murió atropellado en enero de 2013 (ver nota 4). También en el 2013 se confeccionó un petitorio para que se respetaran y reacondicionaran las ciclovías existentes en las avenidas Rademacher y Francisco de Haro, que contó con más de 600 firmas y generó una importante movilización social y mediática.

El 2015 fue un año electoral en la Argentina y Misiones. A nivel nacional, el principal oponente del oficialismo nacional fue Mauricio Macri -después electo presidente de la Nación-, a cargo del gobierno de Buenos Aires y quien hizo de las políticas ciclistas uno de sus principales logros en la gestión porteña. En ese contexto Usa la bici... convocó a todos los candidatos a intendente de Posadas a presentar sus proyectos sobre movilidad ciclista y sacarles un compromiso de proteger y reacondicionar las dos ciclovías con las que contaba la ciudad. Si bien el candidato triunfante no participó de este ciclo; el colectivo mantuvo una reunión con Joaquín Losada, ya en el cargo de intendente, donde manifestó su compromiso con general con promover políticas de fomento de movilidad en bicicleta.

Desde enero de 2015 Posadas cuenta con un sistema de bicicletas públicas gratuito llamado Ecobicis; dependiente del municipio que cuenta con el auspicio del Banco Macro. El sistema ha sido, a su modo, un fruto de la actividad biciactivista Posadeña. Cuenta con dos estaciones emplazadas en el paseo de la costanera del río Paraná y con bicicletas de distintos rodados para incluir a toda la familia. El uso de las biclas está limitado al recorrido marcado en el paseo, que es de una gran belleza escénica y le da un marcado sesgo recreacional en detrimento de la movilidad. Los activistas consultados señalan que el principal déficit del sistema público consiste en no aumentar la cantidad de estaciones para realizar más viajes "de movilidad" por la ciudad.

Ya en el 2016 se constituye el Biciespacio como continuidad de Usa la bici... pero también para representar la incorporación de nuevas personas y nuevos saberes contenidos dentro del biciactivismo. El Biciespacio desarrolla en la actualidad distintos tipos de actividades relacionadas con la bicicleta, como talleres libres de mecánica, espacios de intercambio gratuito de piezas usadas para reparar la bicicleta, actividades de incidencia con las autoridades y ciclos de cine sobre problemáticas urbanas y de movilidad.

En estos tres años de continuidad de activismo ciclista específico y diferenciado de Masa Crítica, primero con Usa la bici... y después con el Biciespacio, la cuestión de lograr una personería jurídica sobrevoló los debates, pero no fue un obstáculo para 
generar actividades ni conseguir recursos. Si bien mantienen un canal abierto con los "políticos", esencialmente en el ámbito local destinado a coyunturas específicas, la experiencia de estas interacciones junto al temor de "quedar pegado" al funcionario los volcaron a privilegiar la autogestión de las actividades utilizando distintas estrategias de fondeo entre las que se destacan las rifas de bicicletas restauradas, el alquiler de las mismas y la venta de bicilicuados ${ }^{7}$. Por otro lado, los costos económicos y personales de mantener una personería jurídica no alcanzan a cubrir las ventajas de gestionar una, ya que el grueso de los recursos con los que cuentan para realizar actividades no son monetarios.

\section{Los ciclistas urbanos de Posadas como generadores de saberes ciudadanos}

Uno de los elementos más utilizados por los movimientos sociales de promoción de la bicicleta para validar sus intervenciones en el campo vial es que son los propios ciclistas a partir de su práctica cotidiana los principales conocedores de las necesidades de infraestructura ciclística y de los riesgos a los que están expuestos. Esta experiencia, sostienen, no se puede traducir en un sistema "racional cartesiano" de movilidad porque involucra aspectos perceptivos, emocionales, físicos y psicológicos que pueden convertirse en un factor crítico para implementar políticas ciclistas exitosas (Sebastián, 2006; Tironi Rodó y Valderrama Barragán, 2017).

Los funcionarios encargados de la movilidad ciclista suelen convocar a los distintos colectivos del pedal a participar en la formulación, implementación para ampliar los consensos sobre estas políticas reconociendo en parte esta premisa. En nuestro país se registran algunas iniciativas del tipo "mesa del ciclista" pero estos mecanismos de consulta y participación adolecen de falta de continuidad y de vinculación con resultados concretos, con algunas excepciones como el PACTO DE MOVILIDAD promovido por la Municipalidad de Rosario en el $2010^{8}$.

La experiencia del Biciespacio de Posadas con los funcionarios locales de tránsito y movilidad no se diferencia mucho de las experiencias del resto de los grupos y asociaciones del país. Aunque la agenda de este colectivo contenga distintos reclamos relacionados con el campo vial posibles de articular con el Estado, como por ejemplo los vinculados a la convivencia entre conductores o la reducción de la huella de carbono; la discusión y diagnóstico sobre infraestructura, más precisamente sobre bicisendas y ciclovías, ocupa una parte central -casi excluyente- de los diálogos con funcionarios o periodistas.

Este énfasis en las ciclovías genera algunas situaciones paradójicas para los colectivos del pedal, como fue el caso del proyecto del Concejal Mutinelli para construir motosendas sobre el trazado de las ciclovías de Avenida Rademacher y Francisco de Haro presentado en mayo de 2017. Según el argumento del edil, el objetivo del proyecto era aprovechar espacio que ya hay creado para las bicicletas, pero que nunca se consolidó debido a la falta de mantenimiento, circunstancia que había sido denunciada por el colectivo Anda la bici... en el año 2013.

Ya estaba ese problema del abandono de las ciclovías, sólo tenían unas líneas amarillas para segregar, y unos avisos en las esquinas (que ya no están). Ese fue un caballito de batalla de la Masa y fue uno de los puntos que los políticos agarraron para acercarse a nosotrxs. En todos estos años no hicieron nada, es más, se cree que los frentistas sacaron los avisos, porque desde la municipalidad dijeron que ellos no habían sido (Entrevista con Paola T.).

El proyecto generó el rechazo inmediato del Biciespacio. Con el lema "Salvemos las bicisendas", generaron una significativa movilización de ciclistas en poco tiempo que incluyó
7. Se trata de la preparación de licuados de fruta en una licuadora fabricada con una bicicleta. Ejemplos de maquinas fabricadas con bicicletas para usos comunitarios se pueden encontrar en Mayapedal (http://www.mayapedal.org).

8. http://www.etr.gov.ar/pacto php. Última visita 29/09/2017. 


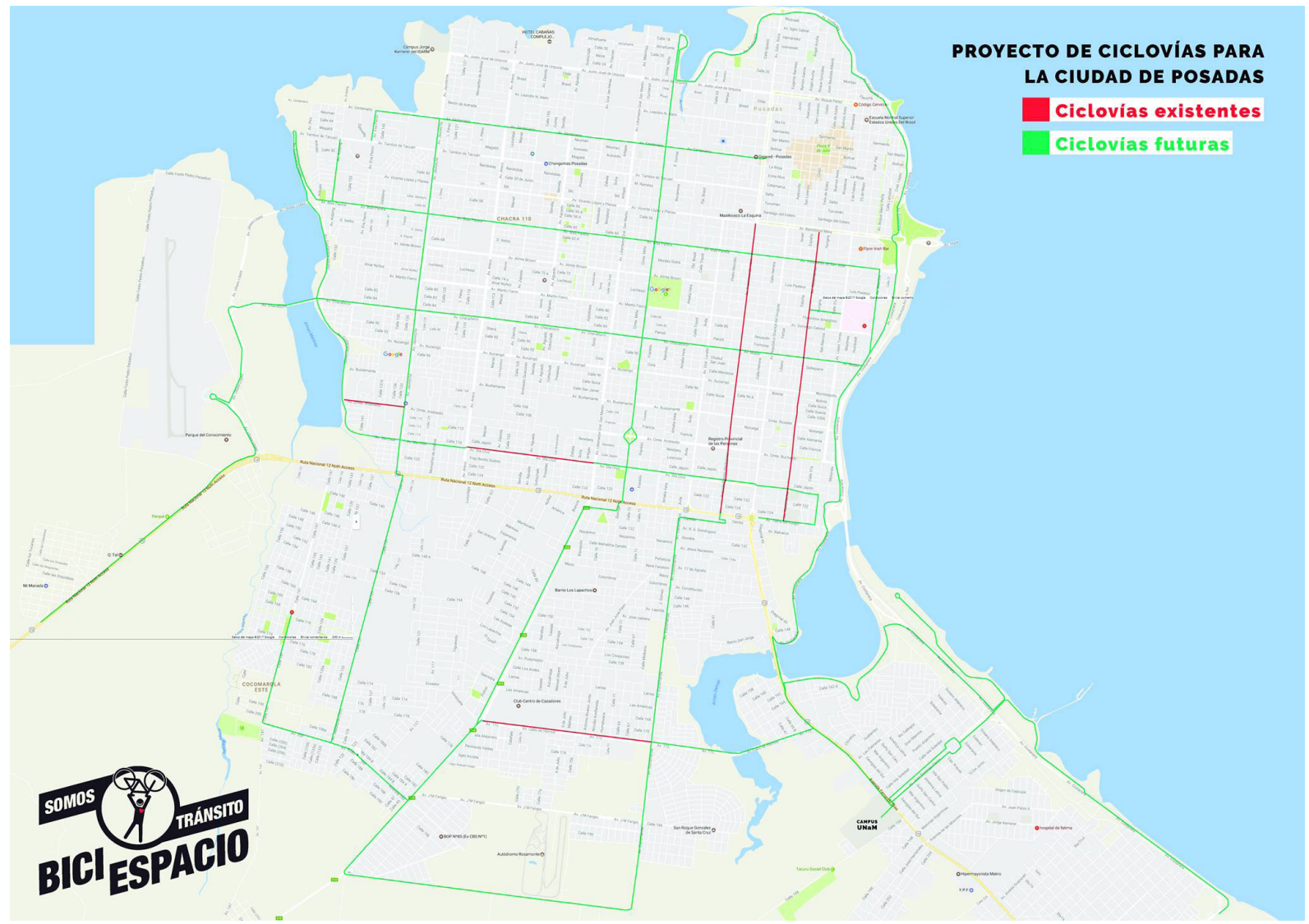

una ronda de medios locales y presencia en el concejo deliberante. Se trataba de defender más que a las ciclovías existentes, el objeto "símbolo" con el que se asocia a los grupos pro bici y aprovechar la coyuntura para proponer un diseño en red de las ciclovías por los principales ejes de la ciudad de Posadas. Esta propuesta, que ellos trazaron "sin estudio de nadie más, basados en nuestra experiencia" (ver Figura 1) es un ejemplo de "saber situado" que pudo ser expresado en un mapa (Ortega Botella; Martinez Novo, 2016).

Las acciones resultaron exitosas en el planteo defensivo. El proyecto de las motosendas se detuvo, se realizaron dos reuniones con la comisión de transporte del concejo de la ciudad y lograron que el concejal se retractara de sus dichos. Pero la movilización de los ciclistas no alcanzó para sostener en el tiempo una agenda concreta de medidas en favor de la bicicleta en Posadas. En parte por estos bloqueos, el Biciespacio retomó lo autogestivo y lanzó la campaña de sensibilización "La convivencia vial es posible" como forma de desmarcarse del pedido de infraestructura ciclista e instalarse en el campo vial con demandas más amplias de ciudadanía "desde la bicicleta". La campaña desarrollará a lo largo del 2017 actividades como bicicleteadas, reparto de calcos y proyecciones.
Figura 1. Propuesta de red de ciclovías para Posadas. Fuente: Biciespacio (2017).

\section{Conclusión}

A lo largo de este artículo visitamos los principales aportes teóricos contemporáneos en movilidad desde las ciencias sociales para poder hacer foco en los grupos, colectivos y asociaciones que promueven la bicicleta como forma de transporte. Para ello utilizamos la categoría de campo vial como un ámbito legítimo donde los distintos actores sociales e institucionales intervienen en y sobre lo vial en una misma red de relaciones de interés e incidencia. 
A continuación repasamos las principales representaciones políticas de la bicicleta, cómo influyeron en la formación de un conjunto de ideas y colectivos sociales a los que definimos a lo largo del artículo como biciactivismo, la emergencia de la Masa Crítica a nivel global y nos detuvimos en los impactos que produjo en nuestro país está "coincidencia organizada".

Cómo vimos, el principal impacto estuvo relacionado con la formación de un nuevo tipo de activismo ciclista, que si bien incluye los reclamos tradicionales de los usuarios de la bicicleta como más infraestructura urbana para la bici; también los excede. La dimensión performativa de la Masa Crítica -muchos, todos juntos y en un recorrido de final abierto- como forma fugaz y contundente de apropiación del espacio público permitió que los ciclistas tuvieran un espacio donde poder manifestarse y protestar por las condiciones de violencia a la que son sometidos en sus pedaleadas cotidianas junto a celebrar la bicicleta como herramienta/objeto de autonomía personal.

La Masa Crítica en nuestro país significó no sólo la formación de una subcultura de la bicicleta, o de una tribu urbana más, sino que propició la constitución de actores con una identidad e intereses específicos en el campo vial. En contra de lo que puede suponerse dado su carácter festivo y anárquico, Masa Crítica hizo viable el paso del "escenificar" a "instituir" a los ciclistas (Escolar, 2000). El surgimiento del colectivo Biciespacio en Posadas expresa la potencialidad del dispositivo Masa como punto de encuentro, facilitador y a su modo organizador de actores sociales constituidos en torno a la movilidad en bicicleta. Como se dijo, muchos de los grupos y asociaciones nucleadas en Argentina en Bici surgieron de las distintas Masas que se desarrollaron y desarrollan en el país.

Los biciactivistas posadeños no se limitan solo a pedir vías ciclistas para la ciudad y conciben a la bicicleta y el ciclismo urbano como algo más que el ir y venir del punto A al B en bici. No se trata solo de la movilidad, ellos plantean una cultura de la bicicleta asociada a valores como la inclusión, el goce del espacio público y el cuidado del ambiente que pueda, a su modo, convertirse en referente antagónico de la cultura del automóvil relacionada con la sociedad de consumo, el individualismo y la violencia.

La difusión de saberes mecánicos, una de las principales actividades del Biciespacio, es producida en esta clave antagónica. Mientras que la mecánica automotriz está cada vez más cerrada al lego -en gran medida por la ampliación de componentes electrónicos principalmente en los motores- la bicicleta sigue manteniendo el "código abierto", como lo definió un activista posadeño. La gran compatibilidad de piezas, sobre todo en bicicletas para usos cotidianos, aumenta la posibilidad de reciclado y de customización y se convierten en objetos políticos contrapuestos al paradigma del consumo capitalista: el automóvil.

Pero el hecho de que funcionarios y lo que podríamos llamar muy genéricamente opinión pública, identifiquen las demandas ciclistas solo con carriles exclusivos para bicicletas produce una encerrona discursiva para los grupos biciactivistas que pretenden instalar una agenda más amplia sobre lo vial. Como sugiere el caso de Posadas y el intento de creación de motosendas; por un lado el biciactivismo no puede desentenderse de esta demanda y del futuro de esa infraestructura que les da reconocimiento de y hacia el campo vial; pero por el otro limita el margen objetivo de las demandas que puedan dirigir al Estado en relación a una agenda más amplia sobre lo vial y el espacio público.

Como muchas ciudades del país, Posadas está a tiempo de evitar los efectos negativos del uso intensivo e individual del automóvil. ¿Lo logrará? ¿Qué papel jugará el biciactivismo posadeño en las transformaciones necesarias para hacer más ciclable la ciudad? El compromiso cotidiano de los biciactivistas que entendieron que la bicicleta no solo es el medio de transporte más saludable y ecológico, sino que es una herramienta central para recuperar de manera democrática los espacios públicos, es parte central de la respuesta a estas preguntas. 


\section{Q Biblografía}

" Agencia Nacional De Seguridad Vial (2012). Plan Estratégico de Seguridad Vial para Motovehículos. Ministerio del Interior y Transporte; Presidencia de la Nación Argentina.

»Augé, M. (2009). Elogio de la bicicleta. Barcelona: Gedisa Editorial

»Ballent, A. (2005). Kilómetro Cero: La Construcción del Universo Simbólico del Camino en la Argentina de los Años Treinta. Boletín del Instituto de Historia Argentina y Americana “Dr. Emilio Ravignani” (27), 108 - 137.

»Bhalla, K. (2013). The Costs of Road Injuries in Latin America 2013. Technical Notes $\mathrm{N}^{\circ}$. IDB-TN-597. Inter-American Development Bank

» BID (2015). Ciclo-inclusión en América Latina y el Caribe. Guía para impulsar su desarrollo. En internet https://publications.iadb.org/handle/11319/6808 (última visita 10/10/2017)

" BID (2016). Iniciativa ciudades emergentes y sostenibles. www.iadb.org/es/ temas/ciudades-emergentes-y-sostenibles/iniciativa-ciudades-emergentes-ysostenibles (última visita 10/10/2017)

»Blanco, J. (2010). Notas sobre la relación transporte-territorio: implicancias para la planificación y una propuesta de agenda. Revista Transporte y Territorio, 3, 172-190. <www.rtt.filo.uba.ar/RTTo0310172.pdf>

»Carlsson, C. (2012). Ruminations of An Accidental Diplomat. En: Carlsson, Chris; Elliott, Lisa; Camarena Adriana (Edits.) (2012) Shift Happens! Critical Mass at 20. San Francisco; Full Enjoyment Books.

"Díaz Vázquez, M. S. (2017). La bicicleta en la movilidad cotidiana: experiencias de mujeres que habitan la Ciudad de México. Revista Transporte y Territorio 16, $112-126$.

»Diz, C. (2016). Objetos Tirados, Objetos Sagrados. Reapropiación tecnológica de la bicicleta. Revista de Antropología Experimental 16, 147-165.

"Escolar, C. (2000). La recuperación del análisis institucional como perspectiva teórica metodológica. En:Topografías de la Investigación, Cora Escolar comp. Buenos Aires, Eudeba. pp. 29-48.

" Gorz, A. (1973). La ideología social del automóvil. Le Sauvage, París, setiembre octubre, 1973.

» Gray, M., Roderick, W. (1978). El libro de la bicicleta. Madrid: H. Blume, Ediciones.

"Gutiérrez, A. (2012). Movilidad y territorio. Herramientas para la Integración Sectorial del Ordenamiento a Escala Barrial. Segundo Seminario de Procesos Urbanos Informales, Bogotá. Setiembre de 2012.

» Gutierrez, A., Reyes, M. (2017). Mujeres entre la libertad y la obligación. Prácticas de movilidad cotidiana en el Gran Buenos Aires. Revista Transporte y Territorio Nº16. Instituto de Geografía - Universidad de Buenos Aires 16, 147-166.

"Herrera Gallo, G. (2006). Calles aptas para todos: el nuevo paradigma de seguridad en el tránsito. De la conducta individual a la accountability del diseño vial en La seguridad en el tránsito en la ciudad de Buenos Aires, Defensoría del Pueblo de la Ciudad de Buenos Aires, Buenos Aires. 
"Instituto Nacional de Estadísticas y Censos (2010). Censo Nacional de Población, Hogares y Viviendas 2010, Buenos Aires.

" Jordi Sánchez, M. (2016). Pobres, Deportistas y Ecologistas. Paradojas, estereotipos y afectos en el ciclismo urbano, un análisis desde las corporeidades. Revista de Antropología Experimental 16, 129-146.

» López León, L. (2016) Pedalear En La Red. Bicicleta, ciudad y movimiento social. Revista de Antropología Experimental 16, 53-69.

»Lorenzi Fernández, E.; Acero, C. (2016). Observación de la calidad de las políticas de movilidad ciclistas desde la participación. Un estudio comparado de Madrid y Sevilla. Revista de Antropología Experimental, 16, 11-22.

"Lorenzi Fernández, E., Ortega Botella, D. (2016). El Reto de la Movilidad Sostenible. Del cuerpo ciclista al espacio urbano y su observación etnográfica. Revista de Antropología Experimental, 16, 1-9.

» Moro, J. (2000) Problemas de agenda y problemas de investigación. En: Topografías de la Investigación Cora Escolar Comp. Buenos Aires: Eudeba, p. 113-140.

»Orellana, D.; Hermida, C. y Osorio, P. (2017). Comprendiendo los patrones de movilidad de ciclistas y peatones. Una síntesis de literatura. Revista Transporte y Territorio 16, 167-183.

" ONU (2011). Plan mundial para el decenio de acción para la seguridad vial 20112020. Ginebra.

"Oppezzo, M. (2008). La relevancia de las organizaciones civiles en la configuración de la inseguridad vial como problema público. V Jornadas de Sociología de la UNLP. Universidad Nacional de La Plata. Facultad de Humanidades y Ciencias de la Educación. Departamento de Sociología, La Plata.

» Organización Panamericana de la Salud (2009). Informe sobre el estado de la seguridad vial en la región de las Américas. Washington, D.C.

" Organización Mundial de la Salud (2004). Informe mundial sobre prevención de los traumatismos causados por el tránsito. Ginebra.

»Pérez López, R. (2017). Vínculos entre la bicicleta utilitaria, recreativa y deportiva: análisis del impacto de los programas Ecobici y Muévete en Bici en la Ciudad de México (2006-2012). Revista Transporte y Territorio 16, 220 - 234.

»Piglia, M. (2014). Autos, rutas y turismo. El automóvil club argentino y el estado. SIGLO XXI. Buenos Aires.

»Planzer, R. (2005). La seguridad vial en la región de América Latina y el Caribe. Situación actual y desafíos. Santiago de Chile: CEPAL.

" Rinaldi, F. (2014) Bicicletas y equidad vial. Hacia nuevas formas de entender el tránsito. Revista Transporte y Territorio 11, 135-139.

» Sanz Alduán, A., Pérez Senderos, R., Fernández, T. (1999). La bicicleta en la ciudad. Manual de políticas y diseño para favorecer el uso de la bicicleta en la ciudad. Ministerio de Fomento, Madrid.

»Sanz Alduán, A. (2005). El viaje de las palabras. Revista SAM 13, Servei de Medi Ambient de la Diputación de Barcelona.

"Sanz Alduán, A. (2009). Viandantes y ciclistas: atravesando la cortina de humo verde. Ingeniería y Territorio. Revista del Colegio de Ingenieros de Caminos y Puertos 86, 50-57. 
»Sanz Alduán, A. (2015). Energía y equidad: el pensamiento radical sobre el transporte de Ivan Illich. Prólogo a ILLICH, I. (2015 [1974]). Energía y equidad. Los límites sociales de la velocidad. Madrid: Díaz \& Pons.

"Sebastián, N. (2006). Uso y promoción de la bicicleta como medio de transporte en la Ciudad Autónoma de Buenos Aires. La seguridad en el tránsito en la ciudad de Buenos Aires, Defensoría del Pueblo de la Ciudad de Buenos Aires, Buenos Aires.

"Sheller, M. y Urry, J. (2006). The New Mobilities Paradigm. Environment and Planning, 38, 207-226.

»Tironi Rodó M., Valderrama Barragán, M. (2017). Transformando la Bicicleta en Laboratorios Móviles. El caso de un self-tracking ciudadano para la ciudad de Santiago. Revista de Antropología Experimental 16, 23-52.

»Villescas Vivancos, F., Pérez Campoy, M., Carbonell Aguirre, O., Nicolás Hernández, M. (2016). Hacia Una Ciudad En Velocípedo: El papel de los niños como agentes esenciales de cambio. Revista de Antropología Experimental 16, 167-181.

»Wright, P., (2010). Imaginarios, símbolos y coreografías viales: una perspectiva Antropológica. II Congreso Iberoamericano de Seguridad Vial, Buenos Aires, 2022 octubre 2010.

\section{Recursos en Internet}

" Activvas Asociación Civil Trabajar Contra la Inseguridad Vial y la Violencia con Acciones Sustentables, www.activvas.org, Última consulta 15/o9/2017

» BID (2014) Iniciativa ciudades emergentes y sostenibles, www.iadb.org/es/ temas/ciudades-emergentes-y-sostenibles/iniciativa-ciudades-emergentes-ysostenibles, 6656.html última consulta 15/09/2017

»Blog Bicivilizados, www.bicivilizados.org, Última consulta 15/o9/2017.

»Blog Ciclofamilia, www.ciclofamilia.wordpress.com, Última consulta 15/og/2017.

»Blog La Fabricicleta, www.lafabricicleta.blogspot.com, Última consulta 15/09/2017.

» Blog Mi diario de bicicleta, http://midiariodebicicleta.blogspot.com.ar, Última consulta 15/09/2017.

» Portal En Bici Seguro, www.enbiciseguro.org.ar, Última consulta 15/09/2017.

»Federación Europea de Ciclismo, www.ecf.com, Última consulta 15/og/2017.

»Fan Page Biciespacio, www.facebook/biciespacio, Última consulta 15/og/2017.

\section{Videos}

"Ted White (1992) The return of the Scorcher https://www.youtube.com/ watch?v=iA66R2sBB_w Última consulta 15/09/2017

» Masa Crítica Posadas - Cuarto aniversario https://www.youtube.com/ watch?v=kevVROUPclg Última consulta 15/o9/2017 


\section{Fernando Rinaldi / fjrinaldi@gmail.com}

Licenciado en Geografía por la Universidad de Buenos Aires. Fue jefe de gabinete de la Secretaría de Derechos Humanos y representante en el consejo consultivo de la Agencia Nacional de Seguridad Vial. Es creador y director del sitio Ciclofamilia y secretario de la Asociación de Ciclistas Urbanos. Es consultor en movilidad activa del Centro de Estudios Desarrollo y Territorio (CEDET) de la Universidad Nacional de San Martín y colabora en la Revista Transporte y Territorio del Instituto de Geografía de la Universidad de Buenos Aires. 